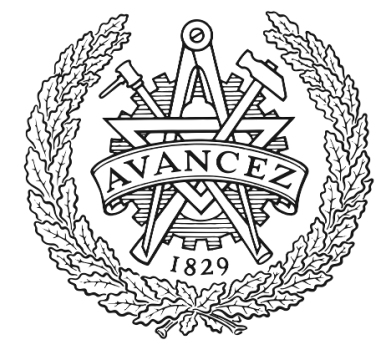

CHALMERS

UNIVERSITY OF TECHNOLOGY

\title{
Qualitative assessment of the bi-stable states in the wake of a finite-width double backward facing step
}

Downloaded from: https://research.chalmers.se, 2023-04-26 11:02 UTC

Citation for the original published paper (version of record):

Rao, A., Zhang, J., Minelli, G. et al (2019). Qualitative assessment of the bi-stable states in the wake of a finite-width double backward

facing step. Journal of Wind Engineering and Industrial Aerodynamics, 186: 241-249.

http://dx.doi.org/10.1016/j.jweia.2019.01.007

N.B. When citing this work, cite the original published paper. 
Short communication

\title{
Qualitative assessment of the bi-stable states in the wake of a finite-width double backward facing step
}

\author{
Anirudh Narayan Rao ${ }^{\mathrm{a}}$, Jie Zhang ${ }^{\mathrm{a}, \text {, }}$, Guglielmo Minelli ${ }^{\mathrm{a}}$, Branislav Basara ${ }^{\mathrm{b}}$, Siniša Krajnović ${ }^{\mathrm{a}, *}$

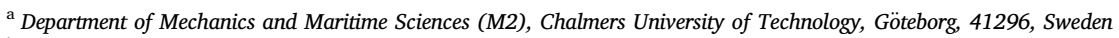 \\ ${ }^{\mathrm{b}}$ AVL List GmbH, Advanced Simulation Technologies, Hans-List-Platz 1, 8020, Graz, Austria
}

\section{A R T I C L E I N F O}

\section{Keywords:}

Wakes

Bi-stability

Backward facing step

Flow control

\begin{abstract}
A B S T R A C T
The flow past a simplified frigate shape model which is analogous to a finite-width double backward facing step is investigated numerically using well-resolved large eddy simulations at $R e \simeq 8 \times 10^{4}$. The geometric configuration of this model permits the occurrence of bi-stable flow states, with an asymmetrical flow topology being observed in the lateral midplane behind each step. In each flow state, the flow at the top step is anti-symmetrical to that observed on the bottom step, and the two flow states are anti-symmetrical to each other. Furthermore, incorporating a base cavity on the top step leads to the suppression of the asymmetrical flow topology on both the steps. The recirculation bubble formed by the downwash at each step is elongated when the base cavity is used, as compared to that observed in either of the two flow states, resulting in a decrease of the drag coefficient. In each of the two flow states, the unequal strength of the streamwise vortices close to the lateral edges on the top step has been identified as the likely cause of the asymmetrical flow on the bottom step.
\end{abstract}

\section{Introduction}

The flow topology of a frigate's flight deck is critical for flight operations; especially for quick take-off and landing of naval helicopters at various headwind conditions (see NATO (2017) guidelines). In this regard, several studies have been performed numerically and experimentally to predict the air-wake of ships and frigates (Wakefield et al. (2002); Syms (2008); Forrest and Owen (2010); Herry (2010); Herry et al. (2011); Kääriä et al. (2013); Van Muijden et al. (2013); Rui et al. (2015); Mora (2014); Mora and Meseguer (2015); Vidales (2016); Orbay and Sezer-Uzol (2016); Gallas et al. (2017); Shi et al. (2017); Crozon et al. (2018) and others). The flow past a frigate wake is analogous to that of a finite-width double-backward facing step (Tinney and Ukeiley (2009)), with the region behind the superstructure and the flight deck forming the top step, and the region behind the stern forming the bottom step. The flow over a double backward-facing step is also applicable to many other engineering applications such as building design, flow around a pick-up truck, etc. Recent experimental investigations have shown that bi-stable flow is probable in the region of the flight deck (Herry (2010); Herry et al. (2011)), with an asymmetrical mean flow topology observed in the lateral midplane of the top step. Their standalone Reynolds-Averaged Navier-Stokes (RANS) prediction for a truncated ship model showed that asymmetrical flow was observed behind the two steps, and that the larger vortex observed on the top step was located on the opposite side behind the bottom step. Furthermore, it was shown that the asymmetrical flow topology (and therefore, the bi-stable flow) observed on the top step was not influenced by placing a splitter plate behind the back step.

The two flow states (arbitrarily named flow state I and flow state II) obtained from the experimental work of Herry et al. (2011) using particle image velocimetry (PIV) are shown in Fig. 1(a) and (b), respectively, along the top step of the ship model at a vertical distance equal to the mid-height of the top step. The two vortices that constitute the asymmetrical flow can be observed, with a larger vortex on one side and a smaller elliptical-shaped vortex on the opposite side of the vertical midplane.

Recent investigations by Zhang et al. (2018) using large eddy simulations (LES) and partially-averaged Navier-Stokes (PANS) equations, showed the existence of the two flow states in the flight deck region of an idealised ship/frigate model (Mora and Meseguer, 2015). Each of the complementary flow states were observed on meshes of different spatial resolution; $\mathrm{M} 1$ and $\mathrm{M} 2$ consisting of $\simeq 10$ million and $\simeq 21.5$ million elements, respectively, with both meshes having adequately resolved the air-wake. The construction of both these meshes was symmetric with respect to the longitudinal midplane of the bluff body. While the

\footnotetext{
* Corresponding authors.

E-mail addresses: zhjie@chalmers.se (J. Zhang), sinisa.krajnovic@chalmers.se (S. Krajnović).
} 


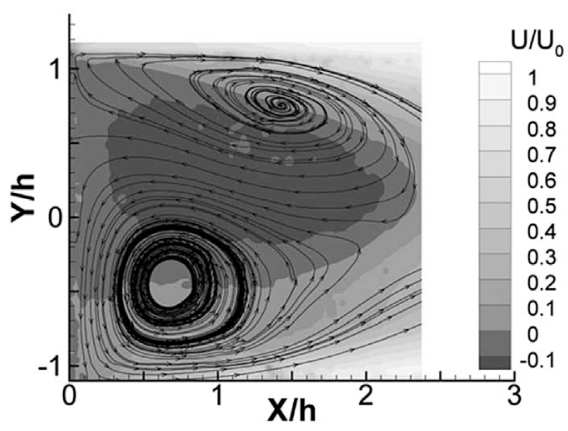

(a)

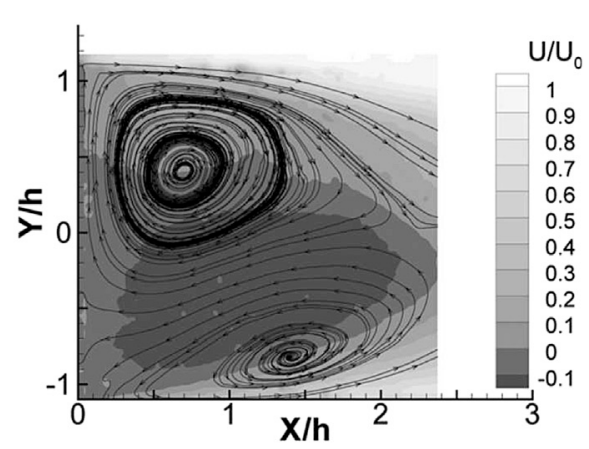

(b)

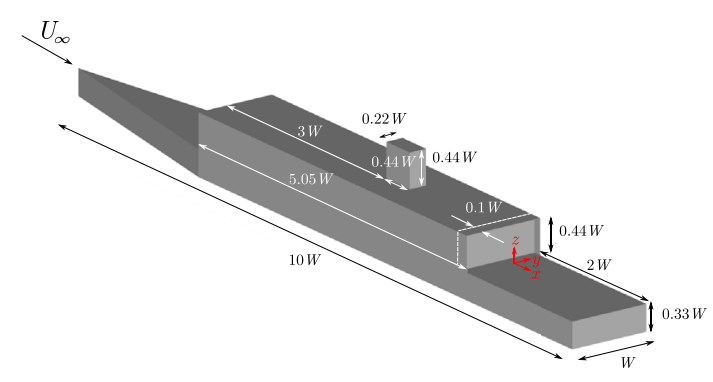

(a)
Fig. 1. Visualisation of the contours of the timeaveraged velocity overlaid with streamlines on the top step the ship model for (a) flow state I and (b) flow state II at $R e=9.75 \times 10^{4}$, based on the height of the top step $(h)$. Flow is from left to right in these images. Image reproduced from Fig. 5 of Herry et al. (2011) (and Fig. 4.19 of Herry (2010)). Permission for reproduction of these images has been obtained from the original publisher - American Society of Mechanical Engineers (ASME).
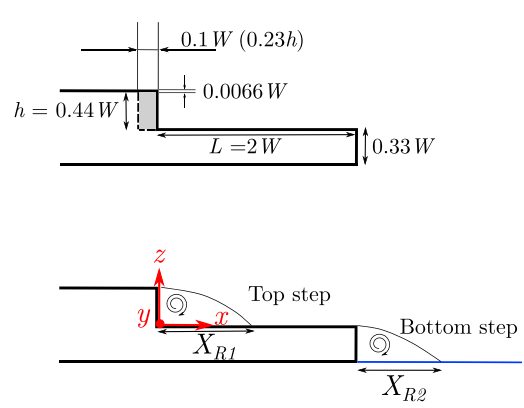

(b)

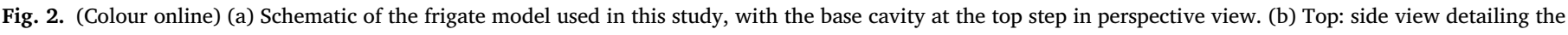

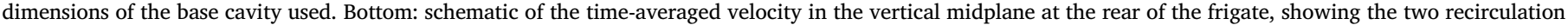
regions. Flow is from left to right in these images.

occurrence of the two stable flow states can be triggered by upstream disturbances, asymmetry to the centreline of the incoming flow or yawing, and geometrical configuration of the body in the experimental investigations; spatial resolution, turbulence modelling approach, numerical schemes used can result in the occurrence of either of the two flow states. Such occurrences have been observed in other bluff body flows such as the idealised car model - the squareback Ahmed body (see Ahmed et al. (1984)), and in the wakes of other parallelepiped bodies (see Grandemange et al. (2015); Grandemange et al. (2013); Bonnavion et al. (2017b) and related studies). However, the occurrence of bi-stability, or the switching phenomenon between the two flow states has not yet been observed numerically, as a result of the short run-times used in the simulations. Longer run-times involving several hundred flow passages would be required to simulate bi-stability, but this is computationally prohibitive.

Previous investigations have largely focussed on the bi-stable behaviour on the top step, and the use of flow control strategies to manipulate the wake (see Wang et al. (2018); Shafer and Ghee (2005); Greenwell and Barrett (2006)). Here, LES are used to show that asymmetrical flow topology exists behind both the top and bottom steps, and that the flow topology on the bottom step is anti-symmetrical to that on the top step; and this flow topology behind the double backward facing step has a complementary flow state. Recent investigations in the wake of a squareback Ahmed body have shown that the presence of a base cavity not only leads to a symmetrical flow topology in the lateral midplane, but also to leads to a reduction in the drag force coefficient on the body (see Lucas et al. (2017); Evrard et al. (2016); Viswanath (1996)). The focus of the current study is primarily on the bi-stable flow characteristics in the wake of a finite-width double backward facing step, and its suppression by incorporating a base cavity on the top step; and the idealised ship model has been used as an example to investigate this fluid dynamics phenomenon. It may be noted the asymmetrical flow topology (and the bi-stability phenomenon) behind squareback bluff bodies remains invariant over a wide range of Reynolds numbers $\left(O\left(10^{3}-10^{6}\right)\right.$ ) (see Volpe et al. (2015); McArthur et al. (2016)). While the upper realm of this limit lies in the operating conditions of ship and truck flows; the lower range of Reynolds number is within the reach of numerical investigations, thereby allowing the simulation of the bi-stable phenomenon using well-resolved LES. The remainder of this "proof of concept" study has been organised as follows: section 2 briefly describes the methodology; the results and the qualitative analysis are presented in section 3 for the two flow states and the base cavity case. This is followed by conclusions in section 4 .

\section{Methodology and validation}

Fig. 2(a) shows the dimensions of a generic ship model (based on the Simplified Frigate Shape (SFS2) model of Syms (2008)) normalised by the width of the ship $(W)$. The length of the heli-deck $(L)$ is equal to twice the width of the superstructure. The inlet velocity is set to $U_{\infty}=8 \mathrm{~ms}^{-1}$ to obtain $R e_{W} \simeq 8 \times 10^{4}$, where $R e_{W}=\rho U_{\infty} W / \mu$ is the Reynolds number based on the width of the ship. Here, $\rho$ is the density and $\mu$ is the dynamic (or absolute) viscosity of the fluid. Fig. 2(b) shows the details of the base cavity on the top step, which extends inwards to a depth of $0.1 \mathrm{~W}$ ( $\simeq 0.23 h$, where $h$ is the height of the top step) into the superstructure. The depth of the base cavity was chosen such that it did not significantly affect the volume of the superstructure/hangar, or cause an extension/protrusion that would minimise the area on the top step (heli-deck), which is critical for the safe flight operations of search and rescue helicopters (see Mora (2014) and references therein). The reduction in the drag coefficient can be observed up to a cavity depth of $\simeq 0.3-0.35$ times the height of an axisymmetric body in freestream (see Fig. 9 of Viswanath (1996)), and a further increase in the depth of the cavity leads to an increase in the drag force on account of the increase in the 
Table 1

Details of the computational grid surface resolution. The number of elements for the two meshes (in millions -M) are shown in the parenthesis. Data reproduced from Zhang et al. (2018).

\begin{tabular}{lll}
\hline Description & M2 $(21.26 \mathrm{M})$ & M1 $(9.93 \mathrm{M})$ \\
\hline$\Delta l_{\text {max }}^{+}$ & $<50$ & $<100$ \\
$\Delta s_{\text {max }}^{+}$ & $<150$ & $<250$ \\
$\Delta l_{\text {mean }}^{+}$ & 30 & 45 \\
$\Delta s_{\text {mean }}^{+}$ & 100 & 120 \\
\hline
\end{tabular}

skin-friction drag on the cavity walls, with the base pressure coefficient remaining constant. The depth of this cavity where the drag minima occurs corresponds to a value of $\simeq 0.132 W-0.154 W$ based on the height of the top step of the ship as the reference length $(0.3 \times 0.44 W=$ $0.132 W ; 0.35 \times 0.44 W=0.154 W$ ). For a squareback Ahmed body, the maximum reduction of the drag coefficient is observed for a cavity depth of $\simeq 0.22 \mathrm{H}-0.27 \mathrm{H}$, where $H$ is the height of the Ahmed body (see Fig. 9(a) of Evrard et al. (2016)). The chosen depth of the base cavity for the ship model is within these prescribed limits, and a reduction in the drag coefficient is predicted to occur for this standalone case based on the above estimates. The thickness of the walls of the cavity or the lip thickness was set to $0.0066 \mathrm{~W}$. The spatial resolution and construction of mesh for the base cavity case is based on mesh M1, and consists of approximately 10.4 million hexahedral elements. For details of the mesh construction, distribution of the elements around the ship and spatial resolution studies the reader is referred to Zhang et al. (2018). The computational domain has a cross-sectional area of $10 \mathrm{~W} \times 10 \mathrm{~W}$, leading to a blockage ratio of less than $1.5 \%$ based on the ratio of the frontal area of the ship to the cross-sectional area of the domain. The inlet boundary is located $10 \mathrm{~W}$ upstream of the frontal edge of the bow, and the outlet boundary is located $25 W$ downstream of the stern. The simulations were started from a quiescent condition, with a uniform velocity assigned to the inlet face of the computational domain, while a homogeneous Neumann boundary condition was assigned at the outlet. A no-slip boundary condition was assigned to the surfaces of the ship and the lower wall, while the top and lateral faces were assigned symmetry boundary conditions. It may be noted that a uniform velocity is used at the inlet face as opposed to a pre-computed oncoming flow mimicking the freestream turbulence, as the latter would be computationally expensive to generate at the Reynolds number considered here (the spatial resolution in the streamwise and spanwise directions on the wall surfaces of the computational domain would have to be similar to that of the bluff body considered). Furthermore, it is highly likely that the smaller fluctuations would be convected quickly, thereby having negligible impact on the flow states observed here (also see Krajnović (2008)).

To adequately resolve the flow in the vicinity of the body, meshes with purely hexahedral elements were constructed. Based on the guidelines of Piomelli and Chasnov (1996) for a well-resolved LES, the first grid point in the wall normal direction needs to be located at $n^{+}<1$, where $n^{+}=\frac{u_{\tau} n}{\nu}$, where, $n$ is the distance of the first cell height from the wall, $u_{\tau}$ is the friction velocity and $v$ is the kinematic viscosity of the fluid; while the spatial resolution in the spanwise and streamwise directions needs to be $\Delta l^{+}=\frac{u_{\tau} \Delta l}{\nu} \simeq 30-50$, and $\Delta s^{+}=\frac{u_{\tau} \Delta s}{\nu} \simeq 50-150$, respectively. Here, $\Delta l$ and $\Delta s$ are the grid spacing in the spanwise and streamwise directions, respectively. Table 1 shows the mean and the maximum values of the above quantities for the two meshes (Also note that for the base cavity case, the mesh construction was identical to mesh M1). The wall normal resolution on the ship model was $n^{+}<1$ for all the three meshes used in this study, with the height of the first cell in the wall-normal direction being set to $0.00025 \mathrm{~W}$. The maximum values of $n^{+}$ were observed at the sharp frontal edges of the ship's triangular bow area.

On account of the triangular bow and the sharp frontal edges of the ship model, the time-step used in these simulations was set to $7.5 \times 10^{-5}$ $\mathrm{s}$ (or non-dimensionalised time-step of 0.04 ), to ensure a CFL number
Table 2

Distances of the recirculation bubbles from the origin at the specified heights. $L$ is the length of the flight deck $(L=2 W)$, and $h$ is the height of the top step ( $h=$ $0.44 W$ ). Data reproduced from Zhang et al. (2018).

\begin{tabular}{llll}
\hline Description & $R e$ & $\begin{array}{l}X_{R 1} \\
(Z / h=0.0667)\end{array}$ & $\begin{array}{l}X_{R 1} \\
(Z / h=0.0067)\end{array}$ \\
\hline LES - Flow state I (M2) & $8 \times 10^{4}$ & $0.462 L$ & $0.504 L$ \\
LES - Flow state II (M1) & $8 \times 10^{4}$ & $0.486 L$ & $0.535 L$ \\
Exp. - Mora and Meseguer (2015) & $8 \times 10^{4}$ & $0.452 L$ & - \\
DES - Forrest and Owen (2010) & $6.58 \times 10^{5}$ & - & $0.450 L$ \\
DES - Forrest and Owen (2010) & $2.26 \times 10^{7}$ & - & $0.490 L$ \\
\hline
\end{tabular}

(Courant-Friedrichs-Lewy condition) less than unity over the domain. The flow field and the forces reported are averaged for three flow passes (for a non-dimensionalised time period $\tau=192$ ) through the domain after an initial transience of one-and-a-half flow passages $(\tau=64)$. The forces converged to better than $2 \%$ between successive flow passages through the domain after the second flow passage (also see Table 3). Fig. 2(b) also shows the flow topology in the vertical midplane of ship, where the flow separates at the top of each step and reattaches at a point downstream. $X_{R 1}$ and $X_{R 2}$ are the reattachment lengths on the top and bottom steps, respectively. The large eddy simulations based on the standard Smagorinsky model (Smagorinsky (1963)) are performed in a commercial finite volume solver, AVL FIRETM (AVL (2014)), and the solver has been used to validate the flow around both simplified and complex geometries (Krajnović et al. (2012), Östh et al. (2014), Minelli et al. (2016), Rao et al. (2018)). It may be noted that the time-integration of the flow is rather short as compared to experimental studies; but the focus of this study is primarily on the qualitative analyses of the two flow states. Nonetheless, the length of the time-integration used here is in line with recent studies on bluff body aerodynamics (see Lucas et al. (2017), Guilmineau et al. (2018), Aljure et al. (2018)).

To ensure that the air-wake was adequately resolved, the comparison between the normalised streamwise velocity profiles $\left(u / U_{\infty}\right)$ at downstream distances of $X / W=0.5$ and $X / W=1$, and at a vertical location of $Z / W=0.44$ from the origin are shown in Fig. 3 for the two flow states. Flow state II (shown by dashed black lines) is transposed to compare directly with flow state I (continuous black lines), and these are compared with the data at identical locations from the numerical study of Syms (2008) (indicated by the continuous blue line) using Lattice-Boltzmann method (LBM), and the experimental data from Aerodynamics Laboratory of the National Research Council, Canada (NRC) at $R e=5.59 \times 10^{5}$ are indicated by the continuous red lines. Despite the larger Reynolds number in the latter study as compared to that of Zhang et al. (2018), the flow asymmetry on the top step is well-predicted at the two streamwise locations, further indicating that the bi-stables states are observed at realistic Reynolds numbers, where ships and other bluff bodies operate.

Lastly, the length of the recirculation zones in the two flow states on the top step is compared with previous experimental studies of Mora and Meseguer (2015) and the detached eddy simulations (DES) of Forrest and Owen (2010) in Table 2. The lengths of the recirculation zones are computed by the zerocrossings of the streamwise velocity at two heights of $Z / h=0.0667$ and $Z / h=0.0067$. While the lengths obtained from the LES are mildly sensitive to the resolution (with a variation of $\simeq 5.5 \%$ ), they are in good agreement with the experimental study of Mora and Meseguer (2015) and the numerical study of Forrest and Owen (2010).

\section{Results and discussions}

Fig. 4(a) and (b) show the contours of the time-averaged velocity overlaid by streamlines at the specified planes on the top and bottom steps in the air-wake of the frigate model. Clearly discernible is the asymmetrical flow topology in both these planes, with one vortex on either side of the vertical midplane. One of the vortices is closer to the 
Table 3

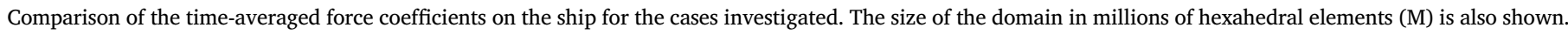

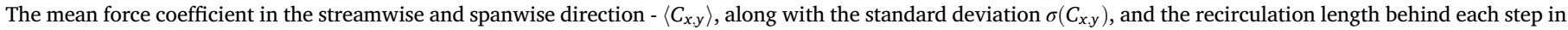
the vertical midplane is presented.

\begin{tabular}{|c|c|c|c|c|c|c|c|}
\hline Description & Size (M) & $\left\langle C_{x}\right\rangle$ & $\sigma\left(C_{x}\right)$ & $\left\langle C_{y}\right\rangle$ & $\sigma\left(C_{y}\right)$ & $X_{R 1} / W$ & $X_{R 2} / W$ \\
\hline Flow state I (M2) & 21.26 & 0.7067 & 0.0175 & -0.0087 & 0.0453 & 1.043 & 0.8031 \\
\hline Flow state II (M1) & 9.93 & 0.7177 & 0.0201 & 0.0034 & 0.0396 & 1.104 & 0.8290 \\
\hline Base Cavity & 10.39 & 0.7041 & 0.0207 & 0.0013 & 0.0363 & 1.139 & 0.8208 \\
\hline
\end{tabular}

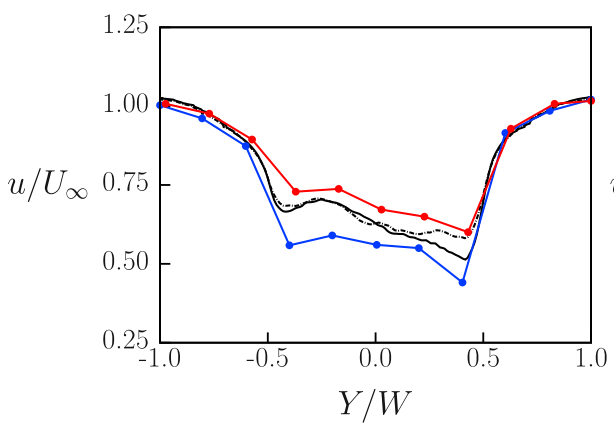

(a)

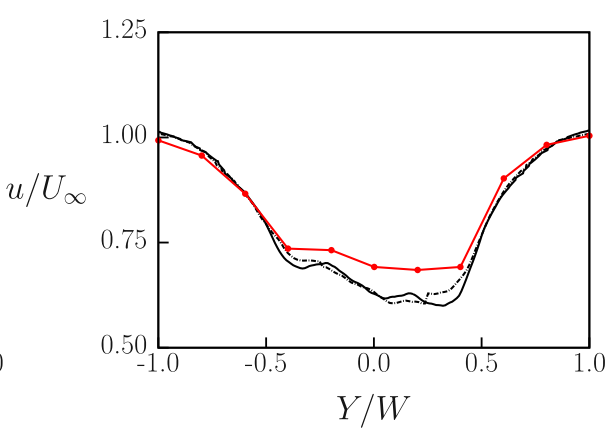

(b)

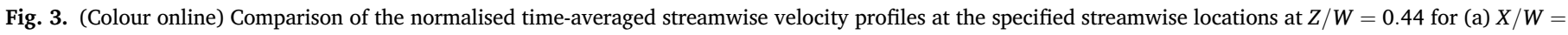
0.5 and (b) $X / W=1$. See text for legend details. Image reproduced from Zhang et al. (2018).

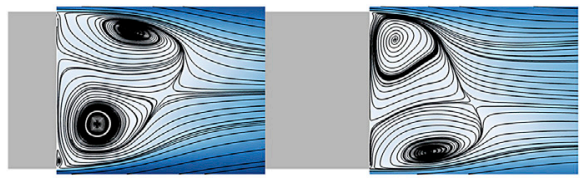

(a)

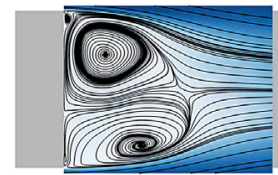

(b)

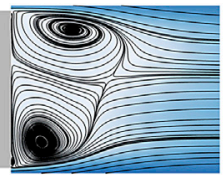

b)
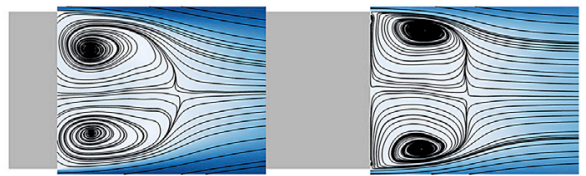

(c)

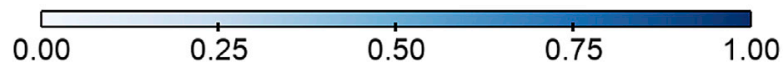

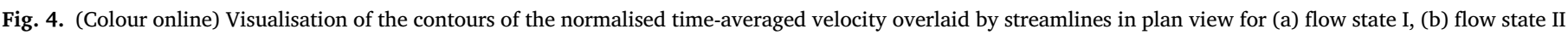

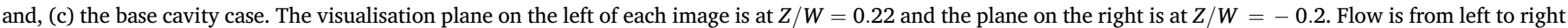
in these images.

base of the ship, while the other is further away from the base, leading to the asymmetry. Furthermore, the flow on the top plane is anti-symmetric to that observed in the bottom plane. Two flow states that are antisymmetrical to each other are observed. Flow state I is observed on the mesh with the finest spatial resolution - M2, flow state II is observed on mesh M1, which has nearly $50 \%$ less elements. Both meshes are adequate to resolve the near-wall flow structures (also see section 2). The differences in the drag and side force coefficient between the two meshes is less than $1.6 \%$ and $1.8 \%$ respectively (see Table 3 ). In flow state I, the vortex further away from the top step of the ship is on the right side, while that behind the bottom step is at the right. This flow topology is anti-symmetric to that observed in flow state II. The height-to-width ratio of the top and bottom steps are 0.44 and $\simeq 0.33$, respectively, and both these ratios independently permit bi-stability in the lateral midplanes (see Fig. 10 (b) of Grandemange et al. (2013)). It may be speculated that a small change in the velocities upstream of the backward facing step may result in the possibility of either of the two flow states being observed when the simulations are started, with the flow "locking" on to this flow state for the remaining duration of the simulations. It may also be recalled that the medium mesh (M1) used here was capable of predicting both flow state I and flow state II when PANS and LES were used, respectively (Zhang et al. (2018)). Nonetheless, the two flow states are physical and have been observed in earlier experimental studies (Herry et al. (2011); Mora (2014)). The two flow states shown in Fig. 1 were obtained after conditionally-averaging (with flow state I being observed $33 \%$ of the duration of their experiment); the same were obtained numerically on two independent simulations (meshes) started from quiescent conditions. The flow topology shown in Fig. 4(a) and (b) on the

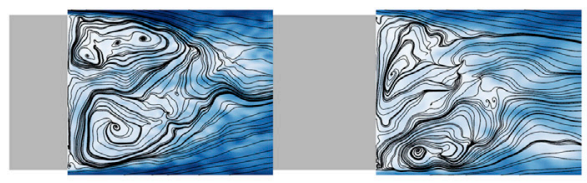

(a)

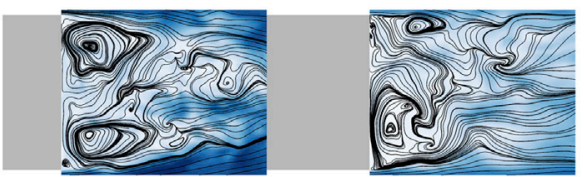

(b)

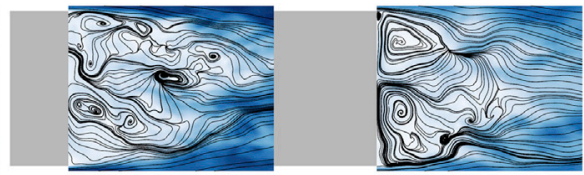

(c)

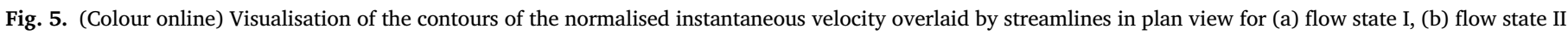

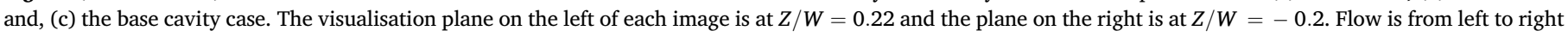
in these images. Contour shading is as per Fig. 4. 


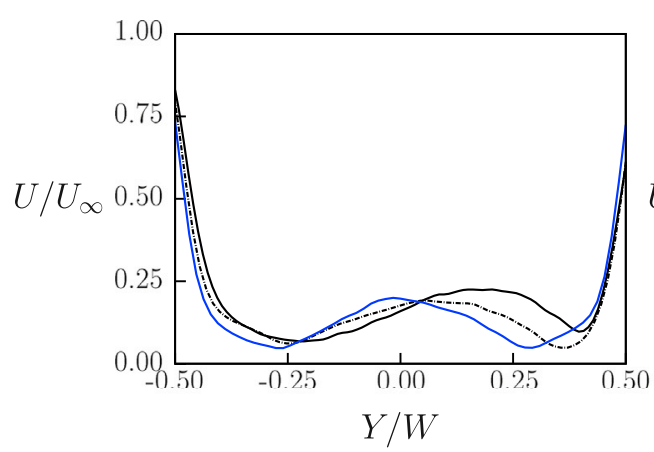

(a)

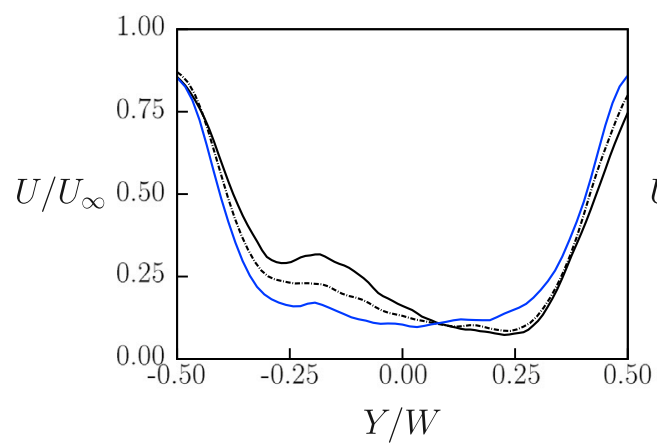

(c)

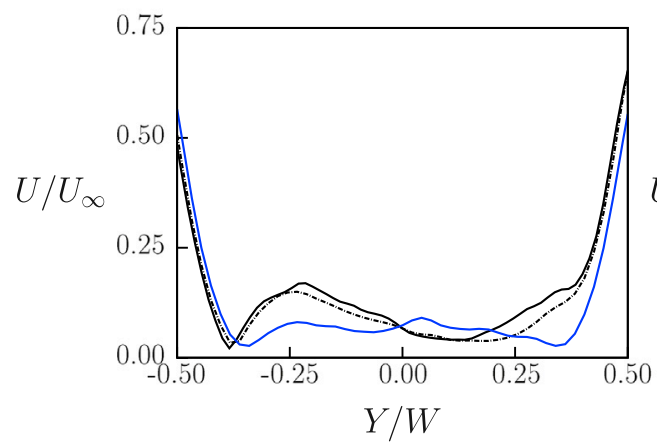

(e)

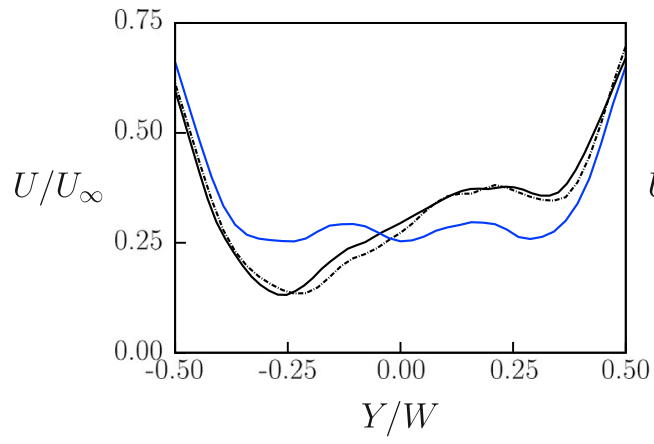

(g)

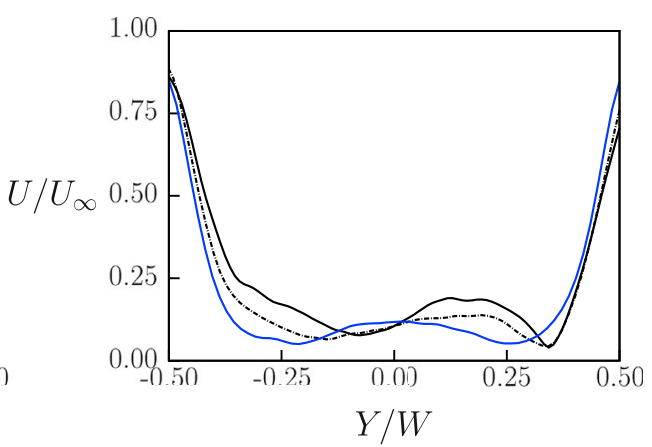

(b)

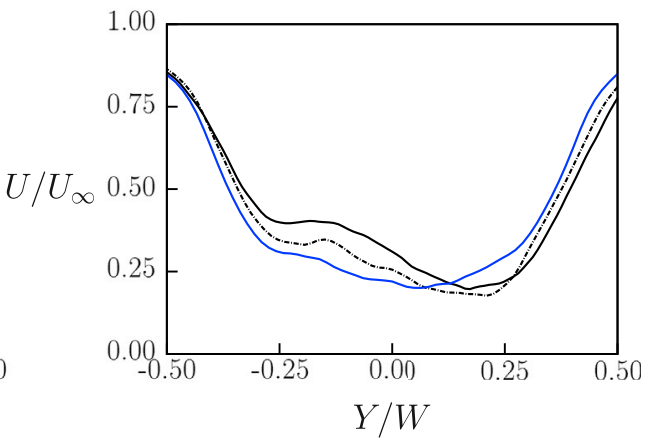

(d)

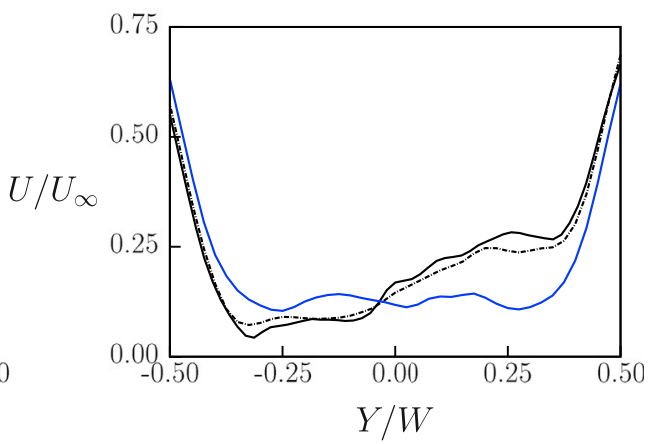

(f)

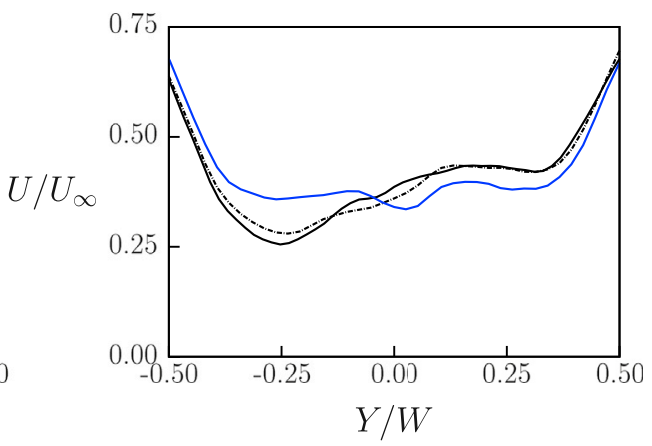

(h)

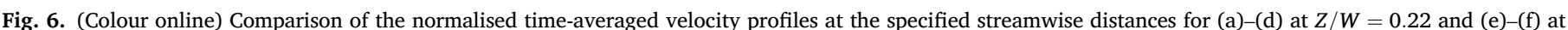

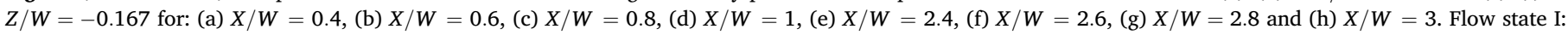
black continuous lines, flow state II: black dashed-dotted lines, and base cavity: blue continuous lines.

top step are qualitatively similar to Fig. 1(a) and (b), respectively.

When the base cavity is used behind the top step, a pair of vortices which are symmetrical about the longitudinal midplane are observed in the lateral plane behind each of the two steps, with the centres of each vortex pair being equidistant from the base as seen in Fig. 4(c) (also see Bonnavion and Cadot (2018)). This is analogous to the flow topology when averaged over the two flow states (see Fig. 5 of Herry et al. (2011)).
Thus, the depth of the cavity chosen is sufficient to suppress the reflection symmetry breaking modes observed in each of the two flow states.

The corresponding (normalised) instantaneous velocity contours for the three cases are shown in Fig. 5(a)-5(c). The predominant vortex structures observed in the instantaneous flows occur in the same locations as those observed in the time-averaged flow (Fig. 4). The instantaneous flow reveals a larger vortex close to the base and a series of 


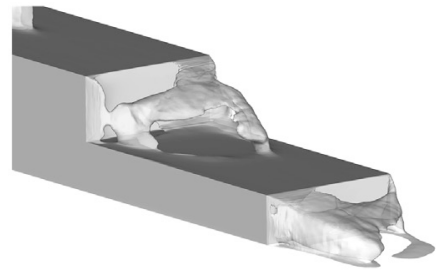

(a)

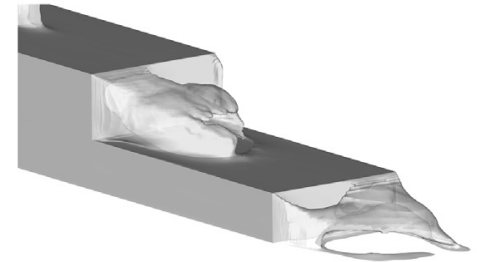

(b)

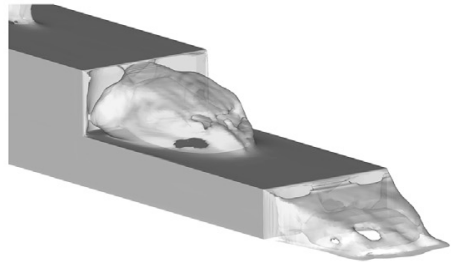

(c)

Fig. 7. (Colour online) Visualisation of the translucent isosurfaces of the time-averaged velocity for (a) flow state I, (b) flow state II and, (c) the base cavity case. Flow is from top left to bottom right in these images.

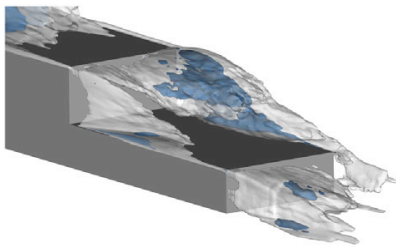

(a)

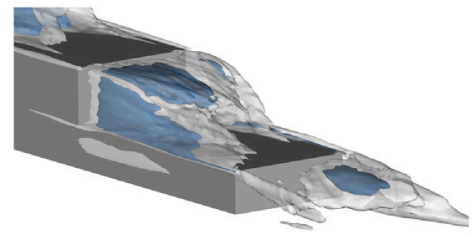

(b)

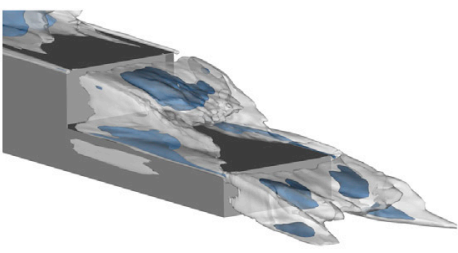

(c)

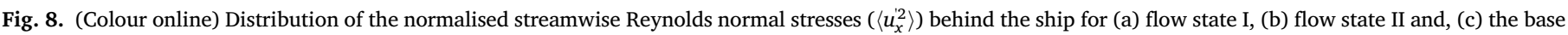
cavity case. Isosurface levels: blue: 0.035 , white: 0.0225 . Flow is from top left to bottom right in these images.

smaller vortices on the opposite side in the two flow states (also see McArthur et al. (2016)); while for the base cavity case, a near-symmetrical distribution of the vortices is observed.

Fig. 6 shows the normalised velocity distribution across the width of the model at the specified streamwise distances for the three cases considered. The velocity profile in flow state II is transposed to compare it with flow state I. The velocity distributions on both the top and bottom steps show an asymmetrical distribution about the $Y=0$ plane, while the velocity distribution for the base cavity case is nearly symmetrical for both the steps. On the top step, a larger flow deficit is observed for $Y /$ $W<0$ up to $X / W \simeq 0.6$ and for $X / W>0.6$, a larger deficit is observed for $Y / W>0$. On the bottom step, a larger flow deficit is observed for $Y$ / $W>0$ up to $X / W \simeq 2.4$ and for $X / W>2.4$, a larger deficit is observed for $Y / W<0$. It may be noted that the velocity distribution is nearly symmetrical for the base cavity case irrespective of the streamwise distance. The comparison of the velocity prediction in the two flow states implicitly shows the good agreement between the two meshes of significantly different spatial resolutions. This furthers gives confidence that the meshes used here are capable of adequately resolving the flow in the near wake.

Shown in Fig. 7(a) and (b) are the isosurfaces of the time-averaged velocity for flow state I and II, respectively. The distribution of the velocity isosurfaces is biased in the region of the vortex farther away from the base. In flow state I, this occurs on the right side on the top step and on the left at the bottom step; while in flow state II, it is anti-symmetric to flow state I. When the base cavity is used, the velocity distribution is symmetrical across the vertical midplane, with the downwash observed being nearly uniform across the span of the base (Fig. 7(c)).

A similar asymmetry is observed in the distribution of the normalised Reynolds normal stresses in the streamwise direction in each of the two

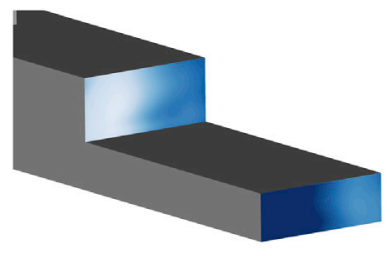

(a)

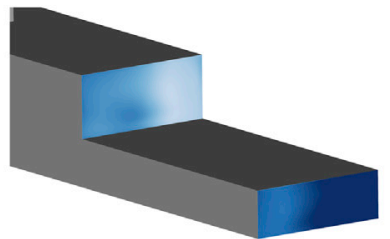

(b)

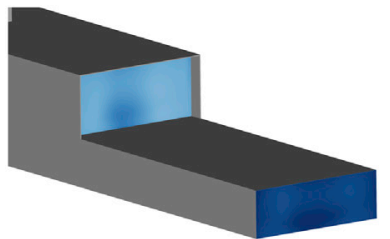

(c)

\begin{tabular}{lllll}
\hline-0.220 & -0.185 & -0.150 & -0.115 & -0.080
\end{tabular}

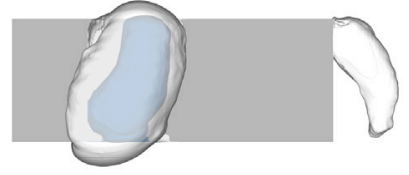

(d)

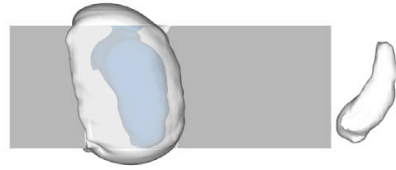

(e)

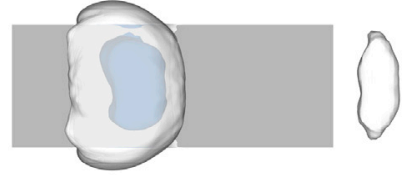

(f)

Fig. 9. (Colour online) Visualisation of the contours of the pressure coefficient $\left(C_{p}\right)$ on the vertical faces of the ship in perspective view in (a)-(c), and visualisation of the translucent isosurfaces of the pressure coefficient in the wake of a ship in plan view in (d)-(f), Isosurface levels: blue: $C_{p}=-0.2$, white: -0.125 . Flow state I - (a), (d), flow state II - (b), (e), and the base cavity case - (c), (f). Flow is from top left to bottom right in images (a)-(c) and from left to right in images (d)-(f). 


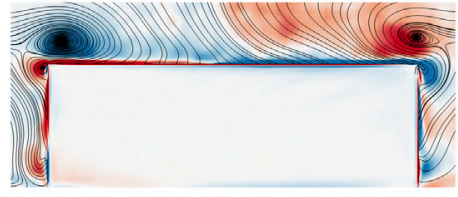

(a)

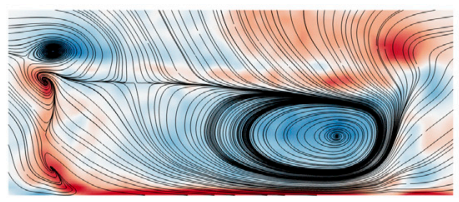

(d)

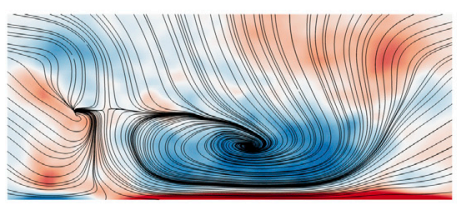

(g)

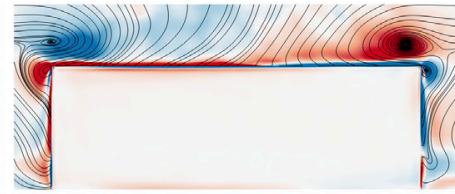

(b)

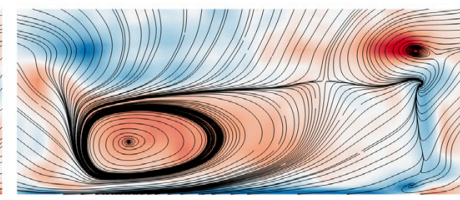

(e)

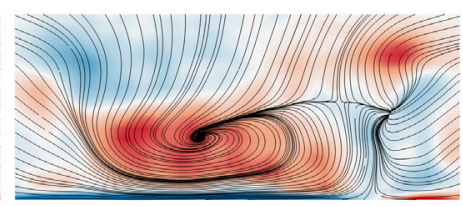

(h)

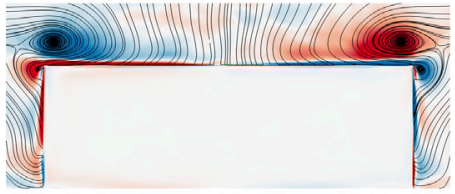

(c)

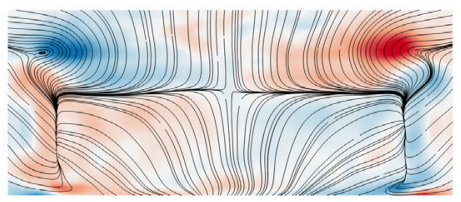

(f)

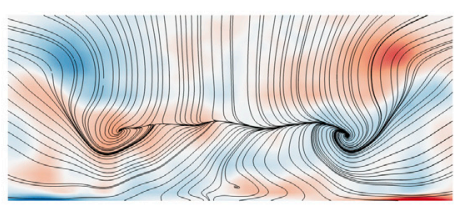

(i)

$\begin{array}{rrrrr}-120 & -60 & 1 & 1 & 120\end{array}$

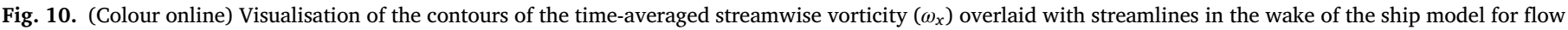

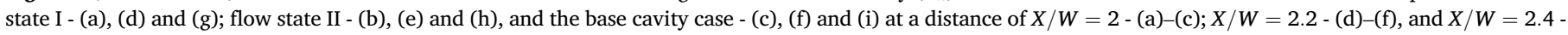

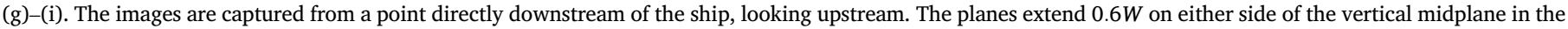
lateral direction, and from $Z / W=-0.33$ to $Z / W=0.167$ in the vertical direction.

flow states (Fig. 8(a) and (b)), with the strongest distribution associated with the (smaller elliptical-shaped) vortex farthest from the base in the time-averaged flow fields of each step, and along the sides. For the model with the base cavity (Fig. 8(c)), the distribution of the stresses are along the upper and side shear layers on both the steps (see Fig. 10 of Lucas et al. (2017) and Fig. 7 of Evrard et al. (2016)). On the top step, the distribution is strongest at the centre, while it is closer to the lateral edges on the bottom step. Nonetheless, the distribution is nearly symmetrical across the vertical midplane when the base cavity is used.

Fig. 9(a)-9(c) show the pressure coefficient $\left(C_{p}=\left(p-p_{\infty}\right) / 0.5 \rho U_{\infty}^{2}\right.$, where $p-p_{\infty}$ is the difference between the local pressure $(p)$ and the reference pressure $\left(p_{\infty}\right)$ ), on the two vertical faces at the rear of the ship. A low pressure region is observed when the larger vortex is closer to the vertical face on each step. In flow state I, the region of low pressure occurs on the left side on the top step, while it occurs on the right side on the bottom step. This distribution is reversed in flow state II. When the base cavity is used, the distribution of the pressure coefficient across the midplane is symmetrical, with lower pressure being observed closer to the two vortex centres. Fig. 9(d)-9(f) show the isosurfaces of the pressure coefficient at the two specified levels in plan view. The flow asymmetry on the two steps are clearly discernible in Fig. 9(d) and (e), while in Fig. 9(f), the distribution of the pressure coefficient is uniform across the width as a result of the base cavity, which leads to the suppression of the asymmetry. The $C_{p}$ isosurface torus is not observed as in the case of the Ahmed body on account of the lack of underbody flow and the upwash; however, the shape of the $C_{p}$ isosurfaces observed on the top step is hemispherical.

It may be recalled that Herry (2010) investigated the genesis of bi-stability, and strategically placed a splitter plate (and a winglet) at various locations at the rear of the ship model to control the strength of the streamwise vortices (see Fig. 4.14 of Herry (2010)). When the splitter plate was placed adjoining to the top step, the occurrence of bi-stability was delayed to higher values of yaw angle. Furthermore, they report that the flow asymmetry on the top step was not influenced when the splitter plate was located behind the bottom step, which indicates that the bi-stable behaviour on the top step is independent of flow asymmetry observed on the bottom step. Here, the flow asymmetry is suppressed on the top step by using a base cavity (also see sections 4.2 and 4.3 of Evrard et al. (2016)), and as a result, the flow asymmetry on the bottom step is also suppressed. The streamwise vortices that are formed close to the lateral edges of the first step are of different strengths on either side of the vertical midplane as seen in Fig. 10.

In each of the two flow states, the streamwise vortex on the side of the larger vortex (and closer to the base) on the top step is larger, and persists further downstream as compared to its counterpart across the vertical midplane (the flow topology on the top step is shown in Fig. 17 of Zhang et al. (2018) and in Yuan et al. (2018)). This asymmetry in the strength of the vortices can be explained based on the isosurfaces of the velocity contours in Fig. 7, where the larger vortex occupies a smaller region behind the top step, thereby allowing a large quantity of flow from the sides of the ship to feed into the streamwise vortex. In flow state I, this larger streamwise vortex occurs on the left side, while in flow state II, it occurs on the right. The downwash on the side of the larger/stronger streamwise vortex occurs further downstream, resulting in an asymmetrical flow topology behind the bottom step (also see Fig. 7). When the base cavity is used, the two streamwise vortices are of equal strength on account of the symmetric flow distribution on the top step, resulting in a symmetrical flow topology behind the bottom step.

Table 3 shows the forces experienced in the streamwise and lateral and the recirculation lengths behind each step for the three cases investigated. The mean drag coefficient $\left(\left\langle C_{x}\right\rangle\right)$ on mesh M1 (flow state II) and mesh M2 (flow state I) are within $1.6 \%$ of each other, and a marginal decrease in the drag coefficient is observed when the base cavity is used. The lengths of the recirculation bubble in the vertical midplane were obtained from the value of the zerocrossing of the streamwise component of the time-averaged velocity at a height of $0.00067 \mathrm{~W}$ from each step (also see Table 2). The length of the recirculation bubble is uniform across $\pm 0.2 W$ of the vertical midplane as seen in Fig. 16 of Zhang et al. (2018), and does not vary significantly from that computed in the vertical midplane. The recirculation lengths, $X_{R 1}$ and $X_{R 2}$ in flow state II are 
Table 4

Comparison of the mean base pressure coefficient on the top step $\left(C_{p, b 1}\right)$ and bottom step $\left(C_{p, b 2}\right)$ for the three cases investigated.

\begin{tabular}{lll}
\hline Description & $C_{p, b 1}$ & $C_{p, b 2}$ \\
\hline Flow state I & -0.1599 & -0.0947 \\
Flow state II & -0.1539 & -0.0868 \\
Base Cavity & -0.1468 & -0.0912 \\
\hline
\end{tabular}

approximately $6 \%$ and $3.2 \%$ longer as compared to flow state I. This difference is perhaps due to the shorter averaging periods considered in the numerical simulations and the inherent asymmetry in the two flow states. When the base cavity is used, the length of the recirculation bubble increases by $\simeq 10 \%$ and $\simeq 2.2 \%$ on the top and bottom steps, respectively, as compared to flow state $\mathrm{I}$, and is $\simeq 6 \%$ longer than the mean values of the two flow states (also see Bonnavion et al. (2017a); Evrard et al. (2016); Wu (1972)).

Table 4 shows the mean base pressure coefficient for the three cases investigated on the top step $\left(C_{p, b 1}\right)$ and bottom step $\left(C_{p, b 2}\right)$. Clearly evident is the increase in the base pressure coefficient on the top step (by $\simeq 9 \%$ ), and a marginal increase on the second step as compared to flow state I, indicating the positive influence of the base cavity on the drag force coefficient. Such devices could be incorporated in frigates leading to the flow stabilisation over the flight deck, which would be beneficial for helicopter flight movements. Furthermore, such devices would also result in fuel savings for the marine and the transportation industry.

\section{Conclusions}

The air-wake of a simplified ship model which is analogous to the flow over a finite-width double backward facing step shows the existence of two stable flow states. On each step, the flow topology was asymmetrical across its width. In each flow state, the flow topology on the top step was found to be anti-symmetrical to that on the bottom step, and the two flow states were anti-symmetrical to each other. The velocity profiles, distribution of the velocity, pressure coefficient and Reynolds stresses were used to qualitatively confirm the existence of the two flow states. By using a base cavity on the top step, asymmetrical flow was suppressed on both the top and the bottom steps, leading to a symmetrical distribution of the aforementioned quantities across the longitudinal midplane of the step. The length of the recirculation bubble in the vertical midplane was elongated when the base cavity was used as compared to that observed in the two flow states without the base cavity. An increase in the mean base pressure coefficient was also observed when the base cavity was used, resulting in a marginal reduction of the drag coefficient. The unequal strength of the streamwise vortices close to the lateral edges on the top step has been identified as the likely cause of the asymmetrical flow topology observed across the bottom step.

\section{Conflict of interest}

The authors declare that they have no conflict of interest.

\section{Acknowledgements}

The authors would like to thank the computational support provided by Chalmers Centre for Computational Science and Engineering (C3SE) and National Supercomputer Centre (NSC), Linköping University provided by the Swedish National Infrastructure for Computing (SNIC). The authors would also like to acknowledge the support and licences provided by AVL GmbH, Austria. Dr. Jie Zhang also acknowledges the financial support from the Area of Advance Energy at Chalmers University of Technology and the Swedish Energy Agency under grant no. 43198-1. The authors would also like to thank the National Research Council of Canada for providing the experimental data of the SFS2 ship model.

\section{References}

Ahmed, S.R., Ramm, G., Faltin, G., 1984. Some salient features of the time-averaged ground vehicle wake. In: SAE Technical Paper, 840300. SAE International.

Aljure, D.E., Calafell, J., Baez, A., Oliva, A., 2018. Flow over a realistic car model: wall modeled large eddy simulations assessment and unsteady effects. J. Wind Eng. Ind. Aerod. 174, 225-240.

AVL, 2014. FIRE CFD Solver Users Guide. AVL GmbH, Graz, Austria.

Bonnavion, G., Cadot, O., 2018. Unstable wake dynamics of rectangular flat-backed bluff bodies with inclination and ground proximity. J. Fluid Mech. 854, 196-232.

Bonnavion, G., Cadot, O., Évrard, A., Herbert, V., Parpais, S., Vigneron, R., Délery, J., 2017a. Effect of a base cavity on the wake modes of the squareback Ahmed body at various ground clearances and application to drag reduction. In: 23rd Congrès Français de Mécanique, Lille, France 28 August 2017 - 1 Septembre 2017, pp. 1-10.

Bonnavion, G., Cadot, O., Évrard, A., Herbert, V., Parpais, S., Vigneron, R., Délery, J., 2017b. On multistabilities of real car's wake. J. Wind Eng. Ind. Aerod. 164, 22-33.

Crozon, C., Steijl, R., Barakos, G.N., 2018. Coupled flight dynamics and CFD demonstration for helicopters in shipborne environment. Aeronaut. J. 122, 42-82.

Evrard, A., Cadot, O., Herbert, V., Ricot, D., Vigneron, R., Délery, J., 2016. Fluid force and symmetry breaking modes of a 3D bluff body with a base cavity. J. Fluid Struct. 61, 99-114.

Forrest, J.S., Owen, I., 2010. An investigation of ship airwakes using detached-eddy simulation. Comput. Fluids 39, 656-673.

Gallas, Q., Lamoureux, M., Monnier, J.C., Gilliot, A., Verbeke, C., Delva, J., 2017. Experimental flow control on a simplified ship helideck. AIAA J. 55, 3356-3370.

Grandemange, M., Cadot, O., Courbois, A., Herbert, V., Ricot, D., Ruiz, T., Vigneron, R., 2015. A study of wake effects on the drag of Ahmed's squareback model at the industrial scale. J. Wind Eng. Ind. Aerod. 145, 282-291.

Grandemange, M., Gohlke, M., Cadot, O., 2013. Bi-stability in the turbulent wake past parallelepiped bodies with various aspect ratios and wall effects. Phys. Fluids 25, 095103.

Greenwell, D., Barrett, R., 2006. In: Inclined Screens for Control of Ship Air Wakes. American Institute of Aeronautics and Astronautics. Chapter Fluid Dynamics and Colocated Conferences, 1-12. 0.

Guilmineau, E., Deng, G.B., Leroyer, A., Queutey, P., Visonneau, M., Wackers, J., 2018. Assessment of hybrid RANS-LES formulations for flow simulation around the Ahmed body. Comput. Fluids 176, 302-319. https://doi.org/10.1016/j.compfluid.2017.01. 005 .

Herry, B., 2010. Aerodynamic study of a 3D backward facing double step applied to safer launch and recovery of helicopters on ships. Theses. Université de Valenciennes et du Hainaut-Cambresis. https://tel.archives-ouvertes.fr/tel-00650410/document.

Herry, B., Keirsbulck, L., Labraga, L., Paquet, J.B., 2011. Flow bistability downstream of three-dimensional double backward facing steps at zero-degree sideslip. J. Fluid Eng. 133, 054501-054501-4.

Kääriä, C.H., Wang, Y., White, M.D., Owen, I., 2013. An experimental technique for evaluating the aerodynamic impact of ship superstructures on helicopter operations. Ocean. Eng. 61, 97-108.

Krajnović, S., 2008. Large eddy simulation of the flow over a three-dimensional hill. Flow, Turbul. Combust. 81, 189-204.

Krajnović, S., Lárusson, R., Basara, B., 2012. Superiority of PANS compared to LES in predicting a rudimentary landing gear flow with affordable meshes. Int. J. Heat Fluid Flow 37, 109-122.

Lucas, J.M., Cadot, O., Herbert, V., Parpais, S., Délery, J., 2017. A numerical investigation of the asymmetric wake mode of a squareback Ahmed body - effect of a base cavity. J. Fluid Mech. 831, 675-697.

McArthur, D., Burton, D., Thompson, M.C., Sheridan, J., 2016. On the near wake of a simplified heavy vehicle. J. Fluid Struct. 66, 293-314.

Minelli, G., Krajnović, S., Basara, B., Noack, B.R., 2016. Numerical investigation of active flow control around a generic truck A-pillar. Flow, Turbul. Combust. 97, 1235-1254.

Mora, R.B., 2014. Experimental investigation of the flow on a Simple frigate shape (SFS). Sci. World J. 1-8.

Mora, R.B., Meseguer, J., 2015. Flow in the near air wake of a modified frigate. Proc. IME G J. Aero. Eng. 229, 1003-1012. https://doi.org/10.1177/0954410014542449.

NATO, 2017. Helicopter Operations from Ships Other than Aircraft Carriers. Technical Report.

Orbay, E., Sezer-Uzol, N., 2016. Computational fluid dynamics simulations of ship airwake with a hovering helicopter rotor. In: Ninth International Conference on Computational Fluid Dynamics (ICCFD9), Istanbul, Turkey, July 11-15, 2016, pp. 1-18.

Östh, J., Noack, B.R., Krajnović, S., Barros, D., Borée, J., 2014. On the need for a nonlinear subscale turbulence term in POD models as exemplified for a highReynolds-number flow over an Ahmed body. J. Fluid Mech. 747, 518-544.

Piomelli, U., Chasnov, J.R., 1996. Large-Eddy Simulations: Theory and Applications. Springer Netherlands, Dordrecht, pp. 269-336.

Rao, A.N., Zhang, J., Minelli, G., Basara, B., Krajnović, S., 2018. An LES investigation of the near-wake flow topology of a simplified heavy vehicle. Flow, Turbul. Combust. https://doi.org/10.1007/s10494-018-9959-6.

Rui, Z., Ji-Li, R., Hai-Xu, L., Peng-Cheng, Z., 2015. Entropy-based detached-eddy simulation of the airwake over a simple frigate shape. Adv. Mech. Eng. 7. https://doi. org $/ 10.1177 / 1687814015616930$.

Shafer, D., Ghee, T., 2005. Active and passive flow control over the flight deck of small naval vessels. In: American Institute of Aeronautics and Astronautics. Chapter Fluid Dynamics and Co-located Conferences, 1-12. 0.

Shi, Y., Xu, Y., Zong, K., Xu, G., 2017. An investigation of coupling ship/rotor flowfield using steady and unsteady rotor methods. Eng. Appl. Comput. Fluid Mech. 11, 417-434. 
Smagorinsky, J., 1963. General circulation experiments with the primitive equations. Mon. Weather Rev. 91, 99.

Syms, G., 2008. Simulation of simplified-frigate airwakes using a Lattice-Boltzmann method. J. Wind Eng. Ind. Aerod. 96, 1197-1206, 5th International Colloquium on Bluff Body Aerodynamics and Applications.

Tinney, C.E., Ukeiley, L.S., 2009. A study of a 3-D double backward-facing step. Exp. Fluid 47, 427-438.

Van Muijden, J., Boelens, O., van der Vorst, J., Gooden, J., 2013. American institute of aeronautics and astronautics. In: Computational Ship Airwake Determination to Support Helicopter-Ship Dynamic Interface Assessment. Fluid Dynamics and Colocated Conferences. AIAA 2013-3078. https://arc.aiaa.org/doi/10.2514/6.20133078.

Vidales, A.F.R., 2016. Air-wake Flow Dynamics on a Simplified Frigate Shape - an Experimental Study by Large-Scale Tomographic PIV. Theses. Delft University of Technology.

Viswanath, P., 1996. Flow management techniques for base and afterbody drag reduction. Prog. Aero. Sci. 32, 79-129.
Volpe, R., Devinant, P., Kourta, A., 2015. Experimental characterization of the unsteady natural wake of the full-scale square back Ahmed body: flow bi-stability and spectral analysis. Exp. Fluid 56, 99.

Wakefield, N.H., Newman, S.J., Wilson, P.A., 2002. Helicopter flight around a ship's superstructure. Proc. Inst. Mech. Eng. 216, 13. Copyright - Copyright Mechanical Engineering Publications, Ltd. 2002; Last updated - 2016-04-23.

Wang, J., Jiang, G., Wang, X., 2018. Effect analysis of the hangar rear edge curvature on the ship airwake. IOP Conf. Ser. Mater. Sci. Eng. 408, 012024.

Wu, T.Y.T., 1972. Cavity and wake flows. Annu. Rev. Fluid Mech. 4, 243-284. https:// doi.org/10.1146/annurev.fl.04.010172.001331.

Yuan, W., Wall, A., Lee, R., 2018. Combined numerical and experimental simulations of unsteady ship airwakes. Comput. Fluids 172, 29-53. https://doi.org/10.1016/j. compfluid.2018.06.006

Zhang, J., Minelli, G., Rao, A., Basara, B., Bensow, R., Krajnović, S., 2018. Comparison of PANS and LES of the flow past a generic ship. Ocean. Eng. 165, 221-236. 